\title{
LA SALUD EN INDOAFROIBEROAMERICA: EL PARADIGMA NECESARIO
}

HEALTH STUDY INDOAFROIBEROAMERICA: PARADIGM NEEDED

Recibido: 22 de Octubre del 2011 | Aceptado: 26 de Noviembre del 2011

ENRIQUE SAFORCADA

(UNIVERSIDAD DE BUENOS AIRES, Buenos Aires, Argentina).

\begin{abstract}
RESUMEN
Los países de Indoafroiberoamérica actúan hoy sobre un conjunto amplio de componentes sociopolíticos: la educación, el desarrollo humano, la seguridad social, la economía, el trabajo, la cultura, etcétera. Ninguno, salvo Uruguay y Venezuela, ha incluido la Salud Pública en sus agendas políticas no obstante haber sido uno de los principales objetivos sobre los que avanzó el Consenso de Washington. La liberación de una nación depende de que el Estado tome las riendas de todas las áreas esenciales para su desarrollo integral. Una de las más importantes es la Salud Pública dado que incide directamente en: a) las posibilidades de desarrollo pleno de las potencialidades de su población y el Desarrollo Nacional; b) la carga de morbilidad y los gastos del Estado en su atención; c) la vulnerabilidad económico polííca y sociocultural del país. Esto no es frecuentemente visualizado por los políticos y políticas ni por la población, tampoco por los profesionales y técnicos de la salud. Este escrito plantea algunas vertientes de reflexión que tienen por objetivo generar líneas de acción académica, política, científica y de las prácticas profesionales. Apunta a incluir la Salud Pública en las agendas políticas de los poderes gubernamentales y de la sociedad.
\end{abstract}

PALABRAS CLAVE: Salud pública; América Latina; procesos de liberación; vulnerabilidad.

\section{ABSTRACT}

The countries of Indoafroiberoamérica act today on an abundant set of socio-political components: education, human development, social security, economy, work, culture, etc. None of then, except for Uruguay and Venezuela, has included the Public Health in its political agendas despite of have been one of the main objectives on which it advanced the Consensus of Washington. The liberation of a nation depends on which the State takes the wheel from all the essential areas for its integral development. One of most important is Public Health since it affects directly: a) the possibilities of total development of the potentialities of its population and the National Development; b) the load of morbidity and the expenses of the State in its attention; $c$ ) the political and socio-cultural and economic vulnerability of the country. This frequently is not visualized by the politicians and policies or by the population, either by the professionals and technicians of health. This writing raises some slopes of political, scientific reflection, that have by objective to generate lines of academic action and of the professional practices. It aims to include Public Health in the political agendas of the governmental powers and the society.

KEY WORDS: Public health; Latin America; processes of liberation; vulnerability.

\footnotetext{
1. Prof. Consulto Titular de Salud Pública y Salud Mental, Facultad de Psicología; Miembro de la Comisión de Maestría en Salud Pública;
} Miembro del Comité de Ética del Instituto de Investigaciones en Salud Pública - Universidad de Buenos Aires.

E-mail: sagar@fibertel.com.ar 
La mayor parte de los países de nuestra región están en un proceso de cambio que se podría caracterizar, obviando las diferencias entre ellos, como procesos de liberación y autodeterminación apoyados en principios básicos de inclusión social, redistribución más equitativa de la renta nacional y respeto de los derechos humanos. Para ello han actuado y actúan sobre un conjunto amplio de componentes sociopolíticos: la educación, el desarrollo humano, la seguridad social, la economía, el trabajo, la cultura, etcétera.

Esto implica una gran oportunidad para generar profundos cambios en el componente de la Salud Pública cuya importancia radica en que el mismo es constituyente de la solidez y potencia de nuestros países. Se debe tener presente que la salud de los pueblos indoafroiberoamericanos ha sido uno de los principales objetivos sobre los que avanzó el Consenso de Washington al imponer el neoliberalismo a través del terrorismo de estado, los golpes económico-financieros hiperinflacionarios y los gobiernos entreguistas (Perkins 2005; Harvey 2007a, 2007b).

Para la liberación de una nación es esencial que su gobierno junto a su pueblo o sea el Estado- tome en sus manos las riendas de todas las áreas esenciales para su desarrollo integral, teniendo en cuenta que no todas ellas implican el mismo quantum de vulnerabilidad nacional cuando las riendas están en manos de corporaciones transnacionales, por lo tanto, de poderes imperiales (Harvey 2007c).

Tal vez, los tres dispositivos que implican la mayor vulnerabilidad para nuestros países son: a) las Fuerzas Armadas y de Seguridad (sobre las cuales ha sobrevolado, frecuentemente se asentó y hoy sigue sobrevolando permanentemente la Escuela de las Américas, del Departamento de Defensa de EE.UU., hoy rebautizada edulcoradamente como Instituto del Hemisferio Occidental para la Cooperación en Seguridad, el mayor centro mundial de desarrollo y capacitación en Terrorismo de Estado, sabotajes y crímenes políticos) b) el Banco Central de cada país junto con el Sistema Económico-Financiero del mismo (objetivos permanentes de la desregulación financiera comenzada en la década de 1970 e impuesta al mundo por parte de EE.UU. e Inglaterra), c) la Salud Pública (desde la década de los 50 del siglo pasado siempre bajo dominio y objetivo de dominio por parte del complejo industrial ${ }^{1}$-corporativo profesional que usufructúa el mercado de la enfermedad $e$, indirectamente, de las universidades donde se forman quienes mantienen activo este mercado). Para quien dude sobre la cuestión de vincular salud 0 Salud Pública y vulnerabilidad nacional le recomiendo la lectura del erudito libro de Sheldon Watts (2000) "Epidemias y poder. Historia, enfermedad, imperialismo".

Con respecto a los dos primeros dispositivos no hace falta ahondar ni dar ejemplos porque los lectores de este escrito en su gran mayoría son indoafroiberoamericanos, ipasamos por muchas dictaduras genocidas y por muchas profundas crisis económicas como para necesitar demostraciones!, en cambio el tema de la Salud Pública en tanto ámbito de vulnerabilidad económico política y sociocultural de un país no es frecuentemente visualizado, ni por las mujeres y los hombres de la política ni por la población, tampoco por los profesionales y técnicos de la salud.

Todos y todas fuimos impactados por ese gigantesco fraude que significó el operativo de marketing desarrollado por un conjunto de tres corporaciones transnacionales de la industria químicofarmacéutica y la Organización Mundial de

\footnotetext{
1 Todas las grandes corporaciones del componente industrial (industria química, electro-electrónica y físico nuclear) de este complejo interesado en la enfermedad de la humanidad son las mismas que están presentes en el complejo industrial-militar denunciado por el General Dwight Eisenhower en 1961 al terminar su último mandato como Presidente de Estados Unidos.
} 
la Salud, secundadas por algunos centros de investigación, corporaciones profesionales y gobiernos para imponer a la humanidad el terror necesario para que nada pudiera obstaculizar la compra masiva del remedio (agente activo: oseltamivir, marca: Tamiflu) y la vacuna que supuestamente actúa con relación al virus de la influenza A (H1N1) o gripe porcina, ocultando su potencial iatrogénico negativo $y$, en el caso de la vacuna, su condición experimental.

Desequilibraron muchos presupuestos municipales y provinciales de salud para concretar esta estafa planetaria, sin trepidar ante el drama que significó y significa hacer entrar a la humanidad en pánico y poner a muchísimas familias y personas individuales ante la disyuntiva de vacunarse y vacunar a sus hijas e hijos sintiendo que era tremendamente peligroso; porque lógicamente, el operativo de marketing fue tan exagerado y carente de reparos que sembró tanto el miedo como la desconfianza.

La pandemia se decretó por parte de la OMS el 11 de junio de 2009 y esta institución anunció el fin de la misma el 10 de agosto de 2010 con una estimación, muy dudosa, de entre 18.000 y 19.000 muertes sobre una población mundial de 6.700 millones de personas; llamar a esto pandemia y haber hecho entrar a la humanidad en pánico pone a la par a la OMS con el Fondo Monetario Internacional en cuanto a condiciones éticas $y$ respetabilidad, también permite ver al servicio de qué poderes trabaja esta institución sacralizada.

El proceso de salud es el proceso de la vida misma y exige el más riguroso respeto como el principal componente de los DDHH, la acabada comprensión de su naturaleza social y la aceptación por evidencia de la espontánea predisposición de los individuos de la especie humana a estar sanos, entendiendo esta condición como la resultante de muy complejos procesos autopoiéticos y homeostáticos que deben ser meticulosamente considerados y no avasallados con artificialidades. Recordemos que ante la enfermedad, la antigua medicina grecorromana hablaba de la vis medicatrix naturae, el poder curador de la naturaleza.

También hay que tener siempre presente que las personas sanas son muchísimas más que las enfermas y entre estas últimas son amplia mayoría las que padecen enfermedades artificiales que las que padecen enfermedades naturales, tal como a mediados del siglo XIX lo manifestaba Rudolf Virchow con relación a las epidemias (Rosen 1985, Waitzkin 2006). Las personas sanas continúan sanas en función de su subjetividad y su comportamiento, de sus estilos de vida y del ambiente en que viven. En primer lugar, no es curando que se logra la mejor condición de salud de una población sino legislando, educando y generando ciudadanía; en segundo lugar, protegiendo y promoviendo la salud 0 , al decir de Aaron Antonovsky (1979, 1987), generando ambientes humanos salutógenos.

Estas reflexiones conducen al planteamiento del problema de los pueblos sojuzgados o colonizados en su salud, tomando en cuenta solo tres aspectos que le son inherentes:

1) El costo, en dinero y sufrimiento, que implica el funcionamiento de un mercado de la enfermedad (Forcades i Vila 2006, Diario Vanguardia 2011, Kaczewer 2003).

2) La enorme obstaculización que este mercado ocasiona al desarrollo nacional por el dinero que drena a lo inconducente y por la hipertrofia que genera en la carga nacional de morbilidad que impide que energías productivas de todo tipo (culturales, científicas, sociales, económicas, etc.) estén disponibles y activas para el 
desenvolvimiento porque están yuguladas por la enfermedad.

3) La enorme ventana de vulnerabilidad que significa para el Estado que la Salud Pública este regida por los lineamientos del neoliberalismo, o sea, en manos de poderes imperiales.

No es esta la oportunidad, por razones lógicas de tiempo, para adentrarnos en cada una de estas manifestaciones 0 consecuencias del problema a fin de discutir caminos de solución o autodefensa, pero ya se gana tiempo y terreno cuando sintéticamente se los visualiza con claridad y eficacia. No pretendo en absoluto estar lográndolo en este escrito, tal vez nos acerquemos a ello con la reflexión que se abra al finalizar su lectura y las alertas que se activen para desnaturalizar lo que hasta ahora está naturalizado.

Al respecto, encuentro importante señalar algunas cuestiones muy básicas como ámbitos donde hay que tomar decisiones que permitan encaminar la Salud Pública hacia la liberación, el bienestar del pueblo y el desarrollo nacional. Una de ellas es el tema de los cuerpos legislativos del Estado en sus tres jurisdicciones (nacional, provincial y municipal) y su producción reguladora que por lenidad o por omisión permite que se genere Enfermedad Pública, entendiendo por tal toda enfermedad que el Estado podría evitar poniendo en juego los recursos de que dispone (económicos, tecnológicos, de conocimientos, humanos, etc.) en el momento o período histórico de que se trate pero que, no obstante, no impide 0 se desentiende de hacerlo 0 protege a quienes o quien la genera.

Para dar solo un ejemplo, tomo una cita del libro de Watts recién mencionado, en que transcribe un pasaje de una obra de Alex Mercer:

Se ha argumentado que ninguna otra industria mata a la gente en la escala de la industria tabacalera, y a diferencia de otras industrias, que deben frenar sus actividades cuando amenazan la salud de los trabajadores y consumidores, esta industria ha podido intensificar sus campañas para persuadir a la gene de consumir sus productos. Históricamente, el interés económico de una pequeña minoría siempre se ha antepuesto a la salud humana [...]. Para definir legalmente si esto es homicidio social $u$ homicidio social culposo, se requeriría un análisis científico realizado por quienes no tengan intereses creados.

Sin lugar a dudas, la carga de morbimortalidad que en nuestros países generan las drogas ilegales, como la cocaína y ni que hablar de la marihuana, resulta un juego de niños en comparación con la que produce el tabaco. Comparar la actitud y toma de decisiones de nuestros Estados con respecto al tabaco con la las que tienen con respecto a las drogas ilegales genera asombro y desazón, reforzando la necesidad de instalar en nuestras reflexiones y trabajos el concepto de Enfermedad Pública como complemento inseparable del de Salud Pública, entendiendo que no tiene sentido hablar 0 actuar con relación a una si no se lo hace con relación a la otra. El tabaco es un proveedor extraordinario de clientela al mercado de la enfermedad y un obstáculo importante al desarrollo nacional dado que la carga de morbimortalidad de un país se correlaciona en proporción inversa con su posibilidad de desarrollo, por razones obvias.

Se podría dar una infinidad de ejemplos como el de los años de vida útil perdidos por accidentes de tránsito, los déficit intelectuales totalmente evitables generados en la infancia, la comida chatarra, la lenidad en la legislación y controles bromatológicos, los accidentes laborales fácilmente evitables, el alto riesgo instalado en el hogar 
argentino y las consiguientes altas tasas de morbimortalidad infantil y de tercera edad por accidentes en la casa, y un largo etcétera.

Otra cuestión básica es el tema de la formación académica de los profesionales y técnicos para el campo de la salud. En la mayor parte de nuestros países tenemos universidades publicas y privadas, lo lógico sería que al menos las públicas formen a sus graduados para las prácticas públicas, que son mucho más complejas que las privadas, de forma tal que el perfil de sus graduados sea funcional al bien común aportando al mantenimiento del pueblo en condiciones de salud sin desatender la enfermedad que no obstante emerja.

Contraviniendo esta lógica, nuestras universidades estatales forman profesionales y técnicos totalmente funcionales al mercado de la enfermedad y a los designios del complejo industrialcorporativo profesional. Esto implica una pieza o elemento esencial para consolidar y hacer potencial 0 actualmente viable eso de la posibilidad de vulnerar a un Estado a través de la salud. En Argentina, el Estado fue vulnerado con lo de la gripe porcina gracias al Ministro de Salud, médico recibido en la Facultad de Medicina de la Universidad Nacional de Tucumán, y por una infinidad de médicos y otros profesionales y técnicos de la salud, también recibidos en universidades públicas, que acompañaron y potenciaron las decisiones del Ministro a lo largo y ancho del país. También ayudaron a manipular la opinión pública al respecto y a desencadenar el miedo en la población de modo que aceptaran la vacuna y otras operaciones de mercado.

Una tercera cuestión básica con respecto a la cual tomar decisiones que permitan encaminar la Salud Pública hacia el bienestar del pueblo y el desarrollo nacional es la educación pre-universitaria, desde los jardines maternales hasta la finalización de la escuela secundaria. Se puede enseñar a leer con la frase "mi mamá me mima" o con la frase "mi mamá me lava bien las manos", da lo mismo en cuanto a este aprendizaje, ahora, con relación a comportamientos y subjetividades posteriores (en la pubertad, la juventud y la vejez) no es para nada igual.

Los hábitos, conocimientos y subjetividades que se desarrollan y adquieren en la niñez temprana y luego en los distintos ciclos de vida hasta llegar al final de la pubertad, en general no se borran más durante el transcurso del vivir. Se puede afirmar sin mucho margen de error que la vejez, en cuanto a condición de salud y calidad de vida, se prefigura en la niñez temprana. ¿Por qué se prescinde y a veces se impide el uso del sistema educativo formal para lograr el desarrollo salubristas de nuestros pueblos?, ¿será ignorancia 0 desatención?, ¿o será sometimiento a los designios 0 exigencias de los usufructuadores del mercado de la enfermedad? Tal vez sea producto de una combinación de todas ellas, pero no cabe duda que la vía de la educación formal es el camino regio hacia el desarrollo salubristas de una nación y el acceso principal al logro de ambientes y estilos de vida salutógenos.

A fin de cuentas, pareciera que es en el campo de la educación donde se presenta la gran posibilidad de lograr que el pueblo junto con los profesionales y técnicos de la salud, mancomunadamente, alcancen a reducir muy significativamente la carga de morbimortalidad de nuestros países, la educación en sus cuatro tramos: jardín maternal, escuela primaria, escuela secundaria y universidad. Los tres primeros tramos son una instancia fundamental en la configuración, por acción o por omisión, de los modos del vivir adulto.

$\mathrm{Si}$ esto pareciera razonable, concluiríamos que la salud de nuestros pueblos depende fundamentalmente de la educación, no de la medicina ni de la 
psicología. Los trabajos de Thomas McKeown (1976, 1990), en los que mostró que es la elevación de la calidad de vida lo que realmente mejoró las condiciones de salud e incrementó la esperanza de vida de la población de Inglaterra, confirman esta conclusión. Al respecto vale una cita de uno de los libros de este autor (1990):

La conclusión más fundamental es que, a excepción de cuando son determinadas en la fecundación o poco después de ella, las enfermedades no son un atributo ineludible de la condición humana, sino el resultado de llevar una vida poco sana y pueden prevenirse si se cambia el modo de vivir.

Suponiendo que se trabajara con eficacia en estas cuestiones que he señalado, junto a varias más que las complementan y las potencian, y alcanzáramos a mejorar significativamente las condiciones de salud de nuestras naciones, nos quedarían varias cosas más por hacer. Entre ellas hay dos de la mayor importancia, pero ambas son o van a resultar muy polémicas.

La primera se refiere a la salud de nuestros pueblos originarios, en cuanto a conocimientos y condiciones. Es mucho lo que se podría hablar con respecto al tema salud y pueblos indígenas de nuestra indoafroiberoamérica. Nuevamente la razón del espacio nos limita, pero quiero hacer hincapié en solo una cuestión que parece relevante, si bien no la voy a desarrollar, solo la enuncio: los universitarios ¿por qué no nos acercamos a aprender de nuestros indios sus profundos y eficaces saberes en salud? Los aymaras, los mayas, los guaraníes, los mapuches y tantas otras etnias de nuestra región tienen formidables conocimientos sobre el proceso de salud, la alimentación y recursos terapéuticos naturales. Por dar un ejemplo: en las villas miserias del Conurbano de Buenos Aires donde residen, en varias de ellas, población criolla y población aymara, mientras se detectan niveles muy altos de desnutrición infantil en las niñas y niños de las familias criollas nunca se ha encontrado un niño 0 niña aymara desnutrido. ¿No llama la atención esta realidad?, a las universidades públicas de Argentina, hasta ahora, no.

La segunda cosa por hacer es más polémica aún. Pero antes de entrar en su consideración es necesario que haga dos aclaraciones: 1) cuando me refiero a la medicina involucro en el concepto y en el campo a la odontología (las dos disciplinas, tal como están desarrolladas, tiene una fuerte base biológica); 2) tomo a la psicología como una de las cuatro disciplinas que manejan las tecnología centrales $^{2}$ en el campo de la salud (las otras tres son las ya mencionadas medicina $y$ odontología, y la antropología).

Efectuadas las aclaraciones vamos al grano: en Indoafroiberoamérica debemos aprovechar nuestra propia solidez intelectual y científica para poner en discusión rigurosa los conocimientos hoy vigente en las ciencias de la salud en la región y cuya vía de entrada también fue parte de los procesos de dominación extranjera. La idea es ir mucho más allá de aquello de medicina, odontología y psicología por evidencia.

El tema es muy amplio y engorroso, una vez más debo recurrir a la síntesis y la parcialidad. También reincidiré en apoyarme en la medicina, por el peso rector que tiene en el ámbito de la salud y también por el poco relieve de la psicología en el mismo lo

\footnotetext{
2. Tecnologías centrales son aquellas que están directamente vinculadas con la solución de los problemas que caracterizan un campo de actividad científico-técnica. Por ejemplo, en el ámbito de la salud, una intervención quirúrgica que tiene por objetivo la cura de una dolencia 0 su atenuación es una tecnología central, una psicoterapia también lo es. Tecnología periférica es toda la actividad administrativa o de maestranza en un hospital, o las actividades que llevan adelante los profesionales de la salud pero que no se vinculan de modo directo con la prevención de la enfermedad (primaria, secundaria 0 terciaria) o la promoción de la salud.
} 
que haría poco eficaz el texto en cuanto a su capacidad de mostración.

Al respecto, tres cuestiones se presentan como relevantes para ponerlas en discusión, pueden parecer inconexas pero a poco que se avance reflexivamente surge su íntimo entrelazamiento. Solo las enuncio como punto de partida o disparador de trabajo posterior:

a. Es en el Renacimiento, cuando comienza a decaer el poder de control social de la iglesia católica en Europa, que la medicina da ese giro copernicano que le impusieron los poderes de la época a través de figuras como la de Paracelso, Fracastoro, Vesalio, van Helmont, Harvey y tantos otros, generalmente muy entrelazados o implicados, para bien o para mal de ellos, con los poderes papales. Este cambio posibilitó que la medicina se convirtiera en la otra institución con un gran poder de coerción sobre la sociedad, control social progresivamente creciente hasta nuestros días. Lo interesante es observar que ambas, religión y medicina moderna, controlan a través del gran disciplinador de las sociedades: el miedo, el temor a la muerte y al sufrimiento.

Al respecto, propongo incursionar en una reflexión, apelando solo al sentido común sobre algo muy sensible porque todos y todas estamos directa 0 indirectamente implicados: la cuestión de la vacunación de los niños y niñas pequeños. Es propio de la especie humana, dentro del reino animal, que las criaturas nacen con mayor inmadurez y su largo período de maduración es el que posibilita el desarrollo de la inteligencia y su capacidad de adaptación a circunstancias y medios tan diferentes.
El proceso ontogenético implica cambios estructurales y tiempos, siendo tan importante un componente como el otro. La criatura humana nace con su sistema psicoinmunoneuroendocrino totalmente inmaduro, de ahí que la leche materna contenga los anticuerpos que el hijo 0 la hija recién nacidos necesita para evolucionar saludablemente a pesar de los microorganismos patógenos presentes en el ambiente. En el proceso natural de exposición a esos agentes nocivos y la progresiva maduración de este sistema, proceso casi totalmente desconocido y seguramente lleno de dinámicas sutiles, la criatura humana va adquiriendo las defensas inmunológicas ante los microorganismos dañinos para su salud y propios de su ambiente. Si el niño forma parte de un ecosistema familiar que promueve bien su desarrollo ¿por qué injuriar ese delicado sistema exponiéndolo artificialmente, desde el nacimiento, a agentes externos patógenos? Todos los que tenemos cierta edad pasamos las eruptivas sin el menor problema, ¿que algunas personas tuvieron complicaciones?, sí, por supuesto, pero no más que las que generan las vacunas, lo que ocurre es que no se hace epidemiología de las consecuencias nocivas de las vacunas 0 al menos no son difundidas públicamente. En mi época de niño, ya para cuando tenía memoria anterógrada suficiente como para recordarlo hoy, recibí mi primera y única vacuna, la antivariólica. Por supuesto que era lo habitual y aclaro que provengo de familia de pediatras, mi padre lo era.

Así como hoy las transnacionales del mercado de la enfermedad están encontrando drogas activas sin un objetivo preciso y luego salen a 
generar la demanda encontrando o "fabricando" dolencias a las cuales aplicarlas, en un pasado no tan lejano se usaron enfermedades benignas en dolencias que había que evitar a todo trance usando vacunas y señalando muy ostensiblemente las poco frecuentes complicaciones en contraste con el daño inminente y muy probable que había que prevenir. De este modo se armó un lucrativo mercado innecesario y peligroso.

Se comenzó con vacunas monovalentes, luego las bivalentes, después la triple y ya estamos en la séptuple. Aparte de lo que el sentido común indica, en cuanto a respetar la naturaleza y sus procesos, hay científicos que hoy afirman que el SIDA podría ser producto de la destrucción progresiva del sistema inmunológico humano por la hipervacunación temprana durante la primera infancia.

No es cuestión de que comencemos a discutir en base a opiniones, la ciencia está para eso, para indagar sobre las dudas y darnos seguridad sobre lo que hacemos y sus efectos. Entonces, ¿por qué no poner bajo investigación científica, en Indoafroiberoamérica, la cuestión de la vacunación infantil en lugar de obedecer ciegamente los mandatos de los laboratorios de los países dominantes?, ¿por qué no contrastar condiciones de vida $y$ enfermedades de la infancia de modo de trazar políticas públicas adecuadas y salutógenas en lugar de vacunar?

Lo curioso es que cuando uno plantea esta cuestión en un ambiente médico la reacción no es la misma que si uno plantea, entre físicos, que la teoría de la relatividad está equivocada. En este último caso se genera una discusión racional apelando a la ciencia, en el anterior la reacción es de escándalo y desaparece la racionalidad en las argumentaciones, un escándalo similar al que se suscita la herejía en un ambiente religioso.

b. Todas las medicinas de las grandes culturas (china, ayurvédica, maya, etc.) son hasta hoy primordialmente higienistas -inclusive la nuestra lo fue hasta el final de la Edad Media-, su objetivo principal es mantener sana a la gente sana y ante la enfermedad prima lo del poder curador de la naturaleza. Algo de esto tuvo la segunda escuela de medicina de Viena allá por el siglo XIX con figuras como Karl Rokitansky (1804-1878), Josef Skoda (1805-1881), Ferdinand von Hebra (1816-1880), Ignaz Philipp Semmelweis (18181865); sobre todo Skoda que propugnaba abstenerse de cualquier intervención terapéutica, dejando al cuerpo recuperarse sólo o a través de dietas apropiadas como tratamiento de elección frente a muchas enfermedades, y Semmelweis que luchó inútilmente para que quienes atendían a las parturientas en los hospitales se lavaran las manos para bajar la tasa de mortalidad por fiebre puerperal tal como él lo había logrado en su sala, mientras que en las otras la mortalidad sobrepasaba el $50 \%$ de las mujeres atendidas.

c. ¿Cuántos errores de sentido común de la medicina se mantuvieron vigentes hasta bien entrado el siglo XX?, por ejemplo las sangrías, discutiendo si el vaso que se abría debía estar cerca del órgano enfermo o lejos, McKeown afirma que esta práctica mató en Europa más gente que todas las epidemias juntas. Otros ejemplo: las ventosas y las cataplasmas con las que nos cocinaron en nuestra infancia a los que hoy pasamos los 70 años o la ablación de las amígdalas que era de rutina ante la primer angina hasta que algún pediatra observó que en algunos casos se asociaba con la aparición de asma a partir de lo cual, aunque tal vez 
fue una asociación errada, liberó a los niños de una práctica que permitía ejercer pura agresión sin la menor base científica.

Ahora, hoy, ¿cuántas prácticas sofisticadas, de gran complejidad técnica, capacidad de injuria y altísimos costos no serán de la misma naturaleza bárbara e inútil que estas que quedaron atrás no hace mucho tiempo?, la complejidad y el sufrimiento no tienen nada que ver con la eficacia terapéutica, el alcohol no desinfecta la herida porque arde, el Merthiolate 0 thiomersal no arde y desinfecta con más eficacia.

La discusión en el siglo XIX y comienzos del XX entre los que apoyaban a Koch y Pasteur y los que se inclinaban por los planteos de Antonio Béchamp no se saldaron en función de rigurosas investigaciones científicas, sino por los mismos métodos con el que se lo hizo a un lado a Semmelweis o se lo desacreditó pedantemente a Skoda hablando de su nihilismo terapéutico.

Nosotros, los países de Indoafroiberoamérica, por todas las razones argüidas y muchas más, necesitamos alcanzar la independencia salubrista apoyándonos para ello en nuestra ciencia, en nuestros recursos y en nuestras realidades socioculturales. Necesitamos despatologizar la medicina, la odontología y la psicología para poder desarrollar un paradigma centrado en una comprensión holística y sistémica del proceso de salud, necesitamos que se imponga un paradigma que lleve a gestionar salud positiva dado que seguir gestionando fundamentalmente enfermedad nos hace, por un lado, más vulnerables $y$, por otro, nos genera más enfermedad que la que se produciría naturalmente.
Finalizo con una cita de Lyall Watson (2000) tomada de su trabajo titulado "La biología del ser: una historia natural de la conciencia":

Yo fui adoctrinado en una visión del mundo, una descripción de la realidad que me parecía muy útil, pero que se ha convertido en alarmantemente estrecha, ya que cierra la posibilidad de que ciertas cosas ocurran simplemente porque no se ajustan a la definición generalizada de cómo funciona el mundo.

\section{REFERENCIAS}

Antonovsky, A. (1979) - Health, Stress and Coping - U.S.A., Jossey-Bass.

Antonovsky, A. (1987) - Unraveling the mystery of health: how people manage stress and stay well - U.S.A., Jossey-Bass.

Diario Vanguardia (2011) - Cura del Cáncer: ¿Bloqueada por las farmacéuticas? (Entrevista con el Nobel de Medicina Richard J. Roberts)

http://www.vanguardia.com.mx/cura_del_can cer:_\%C2\%BFbloqueada_por_las_farmaceu ticas?_(entrevista_con_el_dr._michelakis)731485.html

Forcades i Vila, T. (2006) - Los crímenes de las grandes compañias farmacéuticas Barcelona, Cristianisme i Justícia http://www.cristianismeijusticia.net/quaderns

Harvey, D. (2007a) - Breve historia del neoliberalismo - Madrid, Akal.

Harvey, D. (2007b) - El nuevo imperialismo Madrid, Akal.

Harvey, D. (2007c) - Espacios de esperanza Madrid, Akal.

Kaczewer, J. (2003) - Toxicología del glifosato: riesgos para la salud humana http://www.mamacoca.org/FSMT_sept_2003 les/doc/kaczewer_toxicologia_del_glifosato_ es.htm

McKeown, T. (1976) - The role of medicine. Dream, mirage, or nemesis? - Gran Bretaña, Nuffield Provincial Hospitals Trust (Burgess \& Son).

McKeown, T. (1990) - Los orígenes de las enfermedades humanas - Barcelona, Crítica. 
Perkins, J. (2005) - Confesiones de un gángster económico. La cara oculta del imperialismo americano - Barcelona, Urano.

Rosen, G. (1985) - De la policía médica a la medicina social. Ensayos sobre la historia de la atención a la salud - México, Siglo XXI.

Waitzkin, H. (2006) - Un siglo y medio de olvidos y redescubrimientos: las perdurables contribuciones de Virchow a la medicina social - Medicina Social, Volumen 1, No. 1, junio.

Watts, S. (2000) - Epidemias y poder. Historia, enfermedad, imperialismo - España, Andrés Bello.

Watson, L. (2000) - La biología del ser: una historia natural de la conciencia - En: D. Lorimer (Ed.) - El espíritu de la ciencia. De la experimentación a la experiencia- Barcelona, Kairós. 